\title{
Pharmacokinetic Comparison and Bioequivalence Study of Two Rosuvastatin 20 mg Formulations in Healthy Volunteers
}

\author{
Wen Yao Mak ${ }^{\star}$, Siew Siew Tan², Jia Woei Wong², Siaw Kuen Chin², Ai Boey Lim², Ean Peng Soon², Irene Looi ${ }^{1}$ and Kah Hay Yuen² \\ ${ }^{1}$ Clinical Research Centre, Hospital Seberang Jaya, Jalan Tun Hussein Onn, 13700 Seberang Jaya, Pulau Pinang, Malaysia \\ ${ }^{2}$ Attest Research Sdn Bhd, Room 024, Kompleks EUREKA, Universiti Sains Malaysia, 11800 USM, Pulau Pinang, Malaysia
}

\begin{abstract}
The present study investigated the pharmacokinetics and bioequivalence between two oral rosuvastatin formulations. The study was designed as a two-way crossover, two-period, open-label, randomised, single-dose investigation. Washout period was determined as 14 days due to the long elimination half-life of rosuvastatin. Bioanalysis of plasma samples was performed via liquid chromatography tandem mass spectrometry. The pharmacokinetic parameters were analysed with the non-compartmental model. The study showed AUC $\mathrm{C}_{0-1}$, $\mathrm{AUC}_{0-\infty}$, and $\mathrm{C}_{\max }$ were within the limit of $80.00 \%-125.00 \%$, and concluded the two products were bioequivalent and interchangeable.
\end{abstract}

Keywords: Bioequivalent; Rosuvastatin; Hypercholesterolaemia; Dyslipidaemia; Cardiovascular

\section{Introduction}

Rosuvastatin belongs to a group of compounds collectively named as 3-hydroxy-methylglutaryl coenzyme-A (HMG-CoA) reductase inhibitors, and is used to inhibit cholesterol synthesis in the body [1]. Rosuvastatin is indicated for the treatment of primary hypercholesterolaemia or mixed dyslipidaemia, or as preventive treatment of cardiovascular events [2]. In addition to the earlier reports of lipid-lowering benefits (low-density lipoprotein cholesterol), statins were shown to exhibit pleiotropic effects which is independent of their cholesterol-lowering capability [3].

Rosuvastatin has low absolute bioavailability of approximately $20 \%$. Maximum plasma concentration after oral administration will be reached approximately 3-5 hours post dose, with an elimination half-life of 13 to 21 hours [2,4] The majority of rosuvastatin is excreted in the faeces unchanged (approximately 90\%), with the remaining portion excreted in urine. Rosuvastatin is not extensively metabolized in human, with roughly $10 \%$ of the ingested dose. The principal isoenzyme involved is CYP2C9 [2]. As with other statins, hepatic uptake of rosuvastatin requires the organic anion transporting polypeptides (OATP) membrane transporter. Genetic polymorphism in genes encoding the OATP transporter may potentially impact the uptake capacity, and subsequent hepatic elimination of rosuvastatin [5].

The most commonly reported side effects with the use of rosuvastatin include headache, dizziness, gastrointestinal disturbances and myalgia. Rhabdomyolysis is rare but has been reported in patients [2]. There are evidences which suggest the association of rosuvastatin use with increased risk of diabetes mellitus, but the frequency is dependent of the presence of other risk factors [6,7].

The aim of the current study was to establish bioequivalence between the innovator product (Crestor $20 \mathrm{mg}$ ) and a locally manufactured rosuvastatin film-coated tablet (Hovid-Rosuvastatin $20 \mathrm{mg}$ ) in healthy volunteers.

\section{Method}

\section{Study protocol}

The study protocol was approved by the Malaysian Medical
Research Ethics Committee (MREC) prior to study initiation. In addition, the study was conducted according to the Malaysian Good Clinical Practice guideline, which adhered to the principals of the Declaration of Helsinki. Informed consents from all trial participants were obtained by qualified medical doctors before any trial-related procedures were conducted.

The clinical phase of the study was conducted in the Clinical Trial Unit, Seberang Jaya Hospital (Penang, Malaysia); bioanalysis of samples was performed in the bioanalytical laboratory of the Universiti Sains Malaysia (Penang, Malaysia). Both the clinical and bioanalytical sites were accredited trial sites by the Malaysian National Pharmaceutical Control Bureau.

\section{Participants}

A total of 36 healthy adult male volunteers were recruited into this study after they were considered eligible against the inclusion and exclusion criteria. The inclusion criteria were male between the age of 21 to 55 years old, with body mass index between 18.5 to $29.9 \mathrm{~kg} / \mathrm{m}^{2}$, or fell within $20 \%$ of ideal body weight for height and build according to the Metropolitan Life Insurance Company Standards. The volunteers must also be in good health and physical condition as determined by medical history and laboratory tests prior to study commencement. The laboratory tests included renal and liver function tests (serum creatinine, blood urea nitrogen, total protein count, total albumin, total globulin, total bilirubin, serum glutamic oxaloacetic transaminase (SGOT), and serum glutamate-pyruvate transaminase (SGPT) count), full blood counts (haemoglobin, red cell count, haematocrit, total white cell count and platelet), and fasting blood glucose test. The volunteers

*Corresponding author: Wen Yao Mak, Clinical Research Centre, Hospital Seberang Jaya, Jalan Tun Hussein Onn, 13700 Perai, Pulau Pinang, Malaysia, Tel: 04-3827333 Ext 511; Fax: 04-3902192; E-mail: makwy@crc.gov.my

Received January 04, 2016; Accepted January 27, 2016; Published February 03, 2016

Citation: Mak WY, Tan SS, Wong JW, Chin SK, Lim AB, et al. (2016) Pharmacokinetic Comparison and Bioequivalence Study of Two Rosuvastatin $20 \mathrm{mg}$ Formulations in Healthy Volunteers. J Bioequiv Availab 8: 095-098. doi:10.4172/jbb.1000275

Copyright: $\odot 2016$ Mak WY, et al. This is an open-access article distributed under the terms of the Creative Commons Attribution License, which permits unrestricted use, distribution, and reproduction in any medium, provided the original author and source are credited. 
had shown ability to understand the content of the study information sheet, and had given their consent to participate in the trial.

A number of volunteers were screened but not included into the study as they fell into the exclusion criteria. The exclusion criteria were significant clinical deviation from the norm in physical or clinical determination, a history or suspicion of drug or alcohol abuse or dependence, requirement of tranquilizers, sedatives, chronic medications for hypertension and diabetes, the use of antiplatelet agents, anti-epileptics, opioids, psychotropics, antibiotics, monoamine oxidase (MAO) inhibitors, a history or presence of organ dysfunction, a history or presence of bone-marrow depression, serious blood disorders, cardiac arrhythmias, cardiovascular disease, stroke, bronchospasm, diabetes mellitus, renal diseases, liver diseases, thyrotoxicosis, parkinsonism, benign prostatic hypertrophy, epilepsy or migraine, and malignancy. Volunteers with hypersensitivity towards HMG-CoA inhibitors, participated in other bioequivalence study or donated blood 8 weeks prior to study commencement, unable to comprehend or comply with the study protocol and unable to give informed consent were excluded as well. Volunteers who smoke more than 10 cigarettes a day were excluded.

\section{Study design}

The trial was a two-treatment, two-way, two-period, two-sequence crossover study with single-dose, randomized-sequence and open-label involving adult male volunteers under fasting conditions.

The volunteers were randomly assigned into one of two groups, where the first group received the test formulation during period I of the study and the reference formulation during period II (T-R). Similarly, the second group of volunteers received the reverse order of reference-test formulation ( $\mathrm{R}-\mathrm{T}$ ). The washout period was set at 14 days to ensure adequate elimination of rosuvastatin from the body.

The test product was manufactured by Hovid Berhad, Malaysia (Hovid-Rosuvastatin $20 \mathrm{mg}$ ) while the reference product was produced by IPR Pharmaceuticals Inc, Puerto Rico (Crestor $20 \mathrm{mg}$ ). Common excipient ingredients were used to formulate the test product, which include fillers, binders, disintegrants and lubricants. There was no expected interaction between the active ingredients and excipients which may affect the pharmacokinetics of the rosuvastatin.

The clinical study phase began on March 2015 and was completed successfully on April 2015. Analysis of the biological samples was subsequently completed on May 2015, and the final report was compiled on May 2015.

The volunteers were admitted into the clinical facility one day prior to the study date, and underwent a 10 hour overnight fasting before dosing. One tablet of either the test or reference product was administered to each volunteer by a qualified pharmacist with $240 \mathrm{ml}$ of plain water. Hand and mouth checks were performed by the pharmacist to ensure the dose was administered appropriately. The volunteers were not allowed to consume any water for 1 hour before and 1 hour after dose ingestion (except the water used for administration), in addition to a 4 -hour post dose fasting. All meals and snacks were provided by the clinical facility, and were calorie-counted to ensure consistency. Blood samples were collected by qualified nurse at 0 (predose), 1, 2, 2.5, 3, 3.5, $4,4.5,5,6,8,10,12,24,36,48$ and 72 hours after dosing. A maximum of $5 \%$ deviation from the planned sampling time was allowed before being considered as protocol deviation. The collected blood samples were centrifuged at $3500 \mathrm{rpm}$ for 15 minutes, and were subsequently stored at $-20^{\circ} \mathrm{C}$ until further analysis by the laboratory.
Diletti et al. recommended the use of intrasubject coefficient of variation (intrasubject $\mathrm{CV}$ ) to calculate a sample size of adequate study power [8]. The study power for this investigation was set at $80 \%$. However, the intra-subject CV value for rosuvastatin was not readily available; therefore the number of required volunteers was estimated from previous bioequivalence studies of the same active ingredient. Thota et al. and Zhang et al. reported successful bioequivalence studies with 20 to 40 volunteers $[9,10]$. Hence, it was decided to include 36 volunteers into the study in order to achieve a statistical power of at least $80 \%$, assuming the $\mu \mathrm{T} / \mu \mathrm{R}$ (expected mean values of the test and reference formulations) did not differ more than $5 \%$ and the coefficient of variation was not more than $20 \%$.

\section{Randomisation and blinding}

All volunteers were randomised into one of two groups (TR or RT) by using randomisation software. The study was designed as an openlabel trial where the investigators were aware of the randomisation sequence. The bioanalytical team did not participate in the clinical phase of the study, and therefore was blinded until the analysis was completed.

\section{Tolerability}

All volunteers were closely monitored by study clinicians throughout the trial. Vital signs (blood pressure, pulse rate, respiratory rate and body temperature) were measured at pre-dose, 4-, 10- and 24hour post dose administration. Participants were mandated to report any discomfort or adverse reaction to the investigators throughout the study period.

\section{Drug analysis}

A validated bioanalysis process using liquid chromatographytandem mass spectrometry (LC-MS-MS) was utilised to quantify the plasma levels of rosuvastatin. The system consisted of an Agilent 1200 Series binary pump (Agilent, Waldbronn, Germany), an Agilent 1200 Series degasser (Agilent, Walkdbronn, Germany), an Agilent 1200 Series thermostatted column compartment (Agilent, Waldbronn, Germany) and an Agilent 1200 Series instant pilot (Agilent, Waldbronn, Germany). The MS/MS analyses were completed with an Applied Biosystems API 3200 triple quadrupole mass spectrometer (Applied Biosystems/MDS SCIEX, Ontario, Canada) in positive electrospray ionization mode (ESI). Data acquisition and analysis were performed with Analyst version 1.4.2 (Applied Biosystems/MDS SCIEX, Ontario, Canada).

The chromatography column used in the study was a Phenomenex Luna $5 \mu$ C18 (2) column ( $150 \mathrm{~mm}$ i.d. $\times 2.0 \mathrm{~mm}, 5 \mu \mathrm{m}$ ) (Phenomenex, USA) analytical column, fitted with a SecurityGuard ${ }^{\circ}$ HPLC Guard Cartridge System with a SecurityGuard ${ }^{\circ}$ Cartridges $(\mathrm{C} 18,4 \times 2.0 \mathrm{~mm}$ ID Guard) (Phenomenex, USA). The mobile phase was a mixture of $30 \%$ of $0.2 \%$ formic acid in distilled water and $70 \%$ of methanol. The flow rate was $0.20 \mathrm{ml} / \mathrm{min}$ isocratically. Carbamazepine was used as the internal standard.

\section{PK analysis}

Four pharmacokinetic parameters were analysed in this study: maximum plasma concentration $\left(\mathrm{C}_{\max }\right)$, time to reach maximum plasma concentration $\left(\mathrm{T}_{\mathrm{max}}\right)$, the area under the plasma concentrationtime curve from time zero to the last measurable time $\left(\mathrm{AUC}_{0 . \mathrm{t}}\right)$ and the total area under the plasma concentration-time curve ( $\mathrm{AUC}_{0-\infty}$ ). $\mathrm{C}_{\max }$ and $\mathrm{T}_{\max }$ values could be obtained from the plasma concentrationtime curve directly, whereas the values of $\mathrm{AUC}_{0-\mathrm{t}}$ were derived from 
the trapezoidal formula. $\mathrm{AUC}_{0-\infty}$ values was the sum of $\mathrm{AUC}_{0-\mathrm{t}}$ and $\mathrm{AUC}_{\mathrm{t}-\infty}\left(\mathrm{AUC}_{\mathrm{t}-\infty}\right.$ was derived by dividing the last measurable plasma concentration by the elimination rate constant, $\mathrm{k}_{\mathrm{e}}$ ).

To obtain the value of $k_{e}$, the concentration-time data were first logarithmically $(\ln )$ transformed to allow the fitting of at least three concentration values into the linear regression. The elimination rate constant was then derived from the terminal slope of the line. Half-life of rosuvastatin $\left(t_{1 / 2}\right)$ was calculated from the equation $t_{1 / 2}=\ln 2 / k_{e}[11]$.

\section{Statistical analysis}

The statistical analysis of the pharmacokinetic parameters was performed with EquivTestPK (Statistical Solution, Cork, Ireland). $\mathrm{C}_{\max }, \mathrm{AUC}_{0 \mathrm{-t}}, \mathrm{AUC}_{0-\infty}$ and $\mathrm{T}_{1 / 2}$ were analysed with ANOVA (Analysis of variance) while $\mathrm{T}_{\max }$ was analysed with Wilcoxon Signed Rank Test for paired samples.

Bioequivalence between both formulations was determined by the ratio of the test-versus-reference pharmacokinetic values of $\mathrm{C}_{\max }, \mathrm{AUC}_{0-\mathrm{t}}$ and $\mathrm{AUC}_{0-\infty}$. The $90 \%$ confidence intervals were calculated with two one-sided test procedure where $\alpha=5 \%$ [12]. The range of the confidence interval should fall between $80.00-125.00 \%$ to conclude bioequivalence between both products. The Malaysian Guideline for Conduct of Bioavailability and Bioequivalence Studies allowed a wider range for $\mathrm{C}_{\max }$ when the active pharmaceutical ingredient was categorised as a highly variable drug [13].

\section{Results}

\section{Study participants}

A total of 36 healthy male volunteers were successfully recruited into the study. The demographic characteristics of the subject population were shown in Table 1 .

No dropout or withdrawal was reported in this study, all 36 participants completed the trial. No adverse drug reaction or side effect associated with the ingestion of both test or reference product was reported or observed by study clinicians.

\section{Pharmacokinetic and bioequivalence analysis}

The bioanalytical assay method was validated based on the European Medicines Agency (EMA) guidelines. Several parameters were validated and they included (1) selectivity (2) linearity and range of calibration curve (3) accuracy (4) precision, (5) matrix effect. The sensitivity or limit of quantification as well as the stability of analyte were also determined. The retention time of rosuvastatin and carbamazepine were 4.0 and 3.5 minutes respectively. The method was selective as there was no significant endogenous peak detected from six different sources of blank plasma at the retention times. Accuracy and precision was evaluated through within-day and between-day validation. For within-day validation, the mean measured concentration did not deviate by more than $\pm 9.0 \%$ for all concentrations except lower limit of quantification (LLOQ) and not more than $\pm 18.0 \%$ at LLOQ. For between-day validation, the mean measured concentration did

\begin{tabular}{|l|c|}
\hline \multicolumn{1}{|c|}{ Characteristics } & Value $(n=36)$ \\
\hline Age, $\mathrm{y}$ & 28 \\
Median & 11.0 \\
Interquartile range & \\
\hline Body mass index, $\mathrm{kg} / \mathrm{m}^{2}$ & 23.2 \\
Median & 5.75 \\
Interquartile Range & \\
\hline
\end{tabular}

Table 1: Demographic characteristics of 36 study participants. not deviate by more than $\pm 8.0 \%$ for all other concentrations tested including the LLOQ. The coefficient of variation (CV) values (precision) were less than $12.0 \%$ for both between-day and within-day validation. The matrix effect was within the acceptable limit with the CV values of the internal standard-normalized matrix factor less than $15 \%$. Rosuvastatin concentration was linear over the range of $0.8-80.0 \mathrm{ng} / \mathrm{ml}$ with correlation coefficient of $>0.99$. Stability analysis of rosuvastatin in plasma as well as drug solution showed the compound was stable throughout the duration of the bioanalysis.

The plasma samples of all 36 subjects were analyzed and the data were used for statistical analysis. Figure 1 showed the mean plasma rosuvastatin concentration of the reference product (Crestor $20 \mathrm{mg}$ ) superimposed with that of the test product (Hovid-Rosuvastatin 20 $\mathrm{mg}$ ). The pharmacokinetic parameters of both products were shown in Table 2.

There was no significant statistical difference observed between the logarithmic transformed values of $\mathrm{AUC}_{0-\mathrm{t}}(\mathrm{p}=0.3152), \mathrm{AUC}_{0}$ $\infty(\mathrm{p}=0.2975)$ and $\mathrm{C}_{\max }(\mathrm{p}=0.1348)$ of the two products. The $90 \%$ confidence interval for the above parameters were calculated as between 0.9767-1.1427 ( $\left.\mathrm{AUC}_{0-\mathrm{t}}\right), 0.9800-1.1407\left(\mathrm{AUC}_{0-\infty}\right)$ and 0.9833-1.1945 $\left(\mathrm{C}_{\max }\right)$. Analysis of $\mathrm{T}_{\max }$ also showed no statistical difference $(\mathrm{p}=0.2226)$.

The intrasubject coefficient of variation values were $21.36 \%$, $19.79 \%$ and $22.15 \%$ for $\mathrm{AUC}_{0-\mathrm{t}}, \mathrm{AUC}_{0-\infty}$, and $\mathrm{C}_{\max }$, which were estimated in accordance to the recommendation by Diletti [8]. Based on these values, the study sample size was sufficient to provide a power of more than $80 \%$ at $\alpha=0.05$.

\section{Discussion}

In this study, we investigated and compared the pharmacokinetics

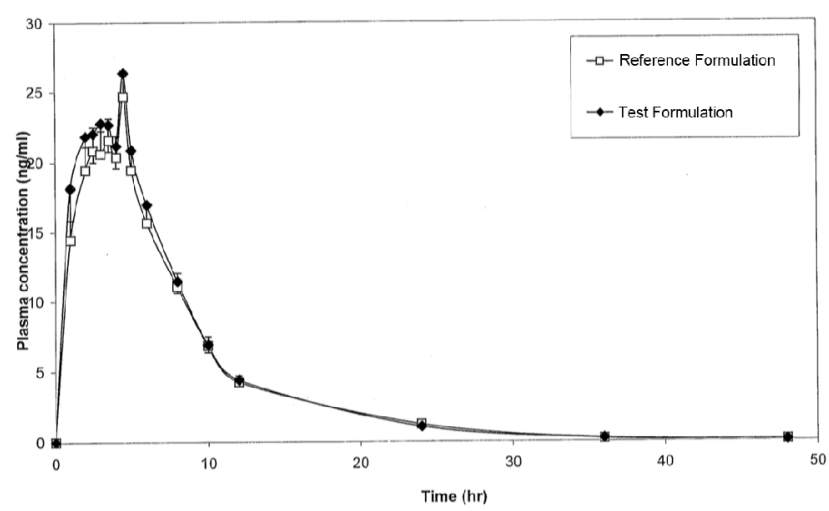

Figure 1: Mean rosuvastatin plasma concentration-time profiles for the test versus reference formulations. Mean \pm SEM

\begin{tabular}{|c|c|c|}
\hline Parameter & Reference Product & Test Product \\
\hline $\begin{array}{l}C_{\max }(\mathrm{ng} / \mathrm{mL}) \\
\text { Mean }(\mathrm{SD})\end{array}$ & $26.2(11.3)$ & $28.4(13.1)$ \\
\hline $\begin{array}{l}\mathrm{AUC}_{0-\mathrm{t}}(\mathrm{ng} / \mathrm{mL}) \\
\text { Mean (SD) }\end{array}$ & $196.9(93.8)$ & $208.3(104.6)$ \\
\hline $\begin{array}{l}\mathrm{AUC}_{0-\infty}(\mathrm{ng} / \mathrm{mL}) \\
\operatorname{Mean}(\mathrm{SD})\end{array}$ & $208.2(95.2)$ & $220.5(107.8)$ \\
\hline $\begin{array}{l}T_{\max }(\mathrm{h}) \\
\text { Median (range) }\end{array}$ & $4.5(7.0)$ & $4.5(5.0)$ \\
\hline $\begin{array}{l}\mathrm{k}_{\mathrm{e}}\left(\mathrm{h}^{-1}\right) \\
\text { Mean }(\mathrm{SD})\end{array}$ & $0.12(0.06)$ & $0.14(0.07)$ \\
\hline $\begin{array}{l}t_{1 / 2}(h) \\
\text { Mean (SD) }\end{array}$ & $6.8(2.8)$ & $6.3(3.2)$ \\
\hline
\end{tabular}

Table 2: The pharmacokinetic parameters of both products. 
Citation: Mak WY, Tan SS, Wong JW, Chin SK, Lim AB, et al. (2016) Pharmacokinetic Comparison and Bioequivalence Study of Two Rosuvastatin 20 mg Formulations in Healthy Volunteers. J Bioequiv Availab 8: 095-098. doi:10.4172/jbb.1000275

of two rosuvastatin tablet formulations in healthy male volunteers. The observed parameters were in agreement with the values reported in the literature. Jung et al. reported similar values in the Korean populations with similar strength, while Vargas et al. reported higher AUC values with their $40 \mathrm{mg}$ tablets but similar $\mathrm{T}_{\max }$ and $\mathrm{k}_{\mathrm{e}}$ values $[14,15]$.

The study was a standard two-formulation, two-period, twosequence crossover trial where the volunteers received both the test and reference products. An adequate washout period was necessary to distinguish the formulation effects from other effects. The EMA guideline recommended a minimum of 5 elimination half-lives between both periods [16]. The $t_{1 / 2}$ of rosuvastatin reported was approximately 19 hours and did not increase at higher doses [2]. The study team had taken consideration of the reported value range, and concluded a 14-day separation between two periods was sufficient for the drug concentration to fall below the lower limit of quantification.

ANOVA analysis was conducted on the pharmacokinetic parameters of $\mathrm{AUC}_{0-\mathrm{t}}, \mathrm{AUC}_{0-\infty}$ and $\mathrm{C}_{\max }$ to evaluate potential difference between separate effects such as formulation, sequence and period effect. None was found to be statistically significant $(\mathrm{p}>0.05)$.

\section{Conclusion}

In conclusion, bioequivalence between the test (Hovid-Rosuvastatin $20 \mathrm{mg}$ ) and reference (Crestor $20 \mathrm{mg}$ ) was concluded based on the acceptance limit of 80.00-125.00\%.

\section{Acknowledgement}

The authors thank all medical staffs at Clinical Research Centre, Hospital Seberang Jaya who contributed to the study.

\section{Declaration of Personal Interest}

Kah Hay Yuen was the advisor to the R\&D department of Hovid Berhad, the manufacturer of the test formulation. Siew Siew Tan, Jia Woei Wong, Siaw Kuen Chin, Ai Boey Lim and Ean Peng Soon were employees of Attest Research Sdn Bhd, an independent research company which is a subsidiary of Hovid Berhad. Wen Yao Mak and Irene Looi did not have any conflict of interest to disclose.

\section{Declaration of Funding Interest}

This study was supported by Hovid Berhad.

\section{References}

1. Kavalipati N, Shah J, Ramakrishan A, Vasnawala H (2015) Pleiotropic effects of statins. Indian J Endocrinol Metab 19: 554-562.
2. (2015) Crestor $20 \mathrm{mg}$ tablets-summary of product characteristics (SPC)-(eMC).

3. Gibson CM, Pride YB, Hochberg CP, Sloan S, Sabatine MS, et al. (2009) Effect of Intensive Statin Therapy on Clinical Outcomes Among Patients Undergoing Percutaneous Coronary Intervention for Acute Coronary Syndrome. J Am Coll Cardiol 54: 2290-2295.

4. Martin PD, Warwick MJ, Dane AL, Hill SJ, Giles PB, et al. (2003) Metabolism excretion, and pharmacokinetics of rosuvastatin in healthy adult male volunteers. Clin Ther 25: 2822-2835.

5. Kalliokoski A, Niemi M (2009) Impact of OATP transporters on pharmacokinetics. Br J Pharmacol 158: 693-705.

6. Carter AA, Gomes T, Camacho X, Juurlink DN, Shah BR, et al. (2013) Risk of incident diabetes among patients treated with statins: population based study. BMJ 346: f2610.

7. Ko DT, Wijeysundera HC, Jackevicius CA, Yousef A, Wang J, et al. (2013) Diabetes mellitus and cardiovascular events in older patients with myocardial infarction prescribed intensive-dose and moderate-dose statins. Circ Cardiovasc Qual Outcomes 6: 315-322.

8. Diletti E, Hauschke D, Steinijans VW (1991) Sample size determination for bioequivalence assessment by means of confidence intervals. Int $\mathrm{J}$ Clin Pharmacol Ther Toxicol 29: 1-8.

9. Thota S, Tippabhotla SK, Khan SM, Nakkawar M (2013) Two-way crossover, bioequivalence study of rosuvastatin tablets $5 \mathrm{mg}$ in healthy, adult, Asian-Indian male volunteers under fasting condition. Int J Pharm Pharm Sci 5: 289-293.

10. Zhang D, Zhang J, Liu X, Wei C, Zhang R, et al. (2011) Validated LC-MS/MS Method for the Determination of Rosuvastatin in Human Plasma: Application to a Bioequivalence Study in Chinese Volunteers. Pharmacol Pharm 2: 341-346.

11. Gibaldi M, Perrier D (1982) Pharmacokinetics, $2^{\text {nd }}$ (Edn) New York: Marcel Dekker 145.

12. Schuirmann DJ (1987) A comparison of the two one-sided tests procedure and the power approach for assessing the equivalence of average bioavailability. $J$ Pharmacokinet Biopharm 15: 657-680.

13. Malaysia Ministry of Health (2000) Malaysian guidelines for the conduct of bioavailability and bioequivalence studies 1-28.

14. Jung JA, Lee SY, Kim JR, Ko JW, Jang SB, et al. (2015) A pharmacokinetic and pharmacodynamic drug interaction between rosuvastatin and valsartan in healthy subjects. Drug Des Devel Ther 9: 745-752.

15. Vargas M, Bustamante C, Villarraga E (2015) Bioequivalence Study of Two Formulations Containing Rosuvastatin $40 \mathrm{Mg}$ Tablets in Healthy Colombians. J Bioequiv Availab 07: 229-232.

16. European Medicines Agency (2010) CPMP/EWP/QWP/1401/98 REV. 1/ $\mathrm{CORR}^{* *}$-Guideline on the investigation of bioequivalence. London 1-27. 